\section{Pontos de venda de alimentos e associação com sobrepeso/obesidade em escolares de Florianópolis, Santa Catarina, Brasil}

\author{
Retail food outlets and the association with \\ overweight/obesity in schoolchildren from \\ Florianópolis, Santa Catarina State, Brazil
}

\author{
Puntos de venta de alimentos y asociación con \\ sobrepeso/obesidad en escolares de Florianópolis, \\ Santa Catarina, Brasil
}

Adriana Filimberti Motter 1

Francisco de Assis Guedes de Vasconcelos 1 Elizabeth Nappi Correa 1

Dalton Francisco de Andrade 1

\footnotetext{
1 Universidade Federal de Santa Catarina Florianópolis, Brasil.

Correspondência A. F. Motter Centro de Ciências da Saúde, Universidade Federal de Santa Catarina. Campus Universitário Trindade, Florianópolis, SC 88040-900, Brasil. adri_motter@hotmail.com
}

\begin{abstract}
The study analyzes retail food outlets and their association with overweight/obesity in schoolchildren from Florianópolis, Santa Catarina State, Brazil. The study used a cross-sectional design with a random sample of 2,506 schoolchildren from public $(n=19)$ and private schools $(n=11)$. Overweight and obesity were classified according to World Health Organization guidelines for 2007, and crude and adjusted analyses were performed using Poisson regression. Prevalence of overweight/obesity was $34.2 \%$. In public schools, $19.6 \%$ of the children were overweight and $13.5 \%$ were obese, as compared to $22.4 \%$ and $11.1 \%$ in private schools. An association was found in the public school system between overweight/obesity and the use of bakeries for food purchases ( $p=0.004)$. In the private school system, children of families that bought groceries at the supermarket showed 26\% less overweight/obesity compared to those who did not $(p=0.003)$. The data show an association between some types of food outlets (supermarkets and bakeries) and prevalence of overweight/obesity in the school-age population.
\end{abstract}

Overweight; Pediatric Obesity; Feeding

\section{Resumo}

O estudo descreve os pontos de venda de alimentos e sua associação com sobrepeso/obesidade em escolares de Florianópolis, Santa Catarina, Brasil. Desenho transversal com amostra probabilística de 2.506 escolares de escolas públicas $(n=19) e$ privadas ( $n=11)$. O sobrepeso/obesidade foi classificado pela referência da Organização Mundial da Saúde de 2007. Foram realizadas análises brutas e ajustadas por meio de regressão de Poisson. A prevalência de sobrepeso/obesidade foi de $34,2 \%$. Na rede pública, foram verificados $19,6 \%$ de sobrepeso e 13,5\% de obesidade. Na rede privada, observaram-se 22,4\% de sobrepeso e 11,1\% de obesidade. $\mathrm{Na}$ rede pública, foi encontrada associação entre sobrepeso/obesidade e utilização da padaria ( $p=$ 0,004 ). Na rede privada, observou-se que os escolares de famílias que utilizaram o supermercado apresentaram 26\% menos de sobrepeso/obesidade do que os escolares que não utilizam esses pontos de venda de alimentos ( $p=0,003)$. Os dados encontrados evidenciam a existência de associação entre a utilização de alguns tipos de pontos de venda de alimentos (supermercado e padaria) e a prevalência de sobrepeso/obesidade na população escolar.

Sobrepeso; Obesidade Pediátrica; Alimentação 


\section{Introdução}

O sobrepeso/obesidade iniciados na infância e na adolescência podem permanecer na fase adulta e levar ao desenvolvimento de doenças crônicas não transmissíveis (DCNT), como diabetes tipo 2 e doenças cardiovasculares, reduzindo a qualidade e a expectativa de vida 1 .

Dados da Organização Mundial da Saúde (OMS) indicam que, na população mundial, $10 \%$ das crianças e dos adolescentes entre 5 e 17 anos de idade apresentam excesso de massa corporal, e 2 a $3 \%$ delas são obesas ${ }^{2}$. No Brasil, dados da Pesquisa de Orçamentos Familiares (POF) de 2008-2009 revelaram que uma em cada três crianças de 5-9 anos de idade (33,5\%) apresentava sobrepeso e $14,3 \%$ obesidade, e, entre os adolescentes de 10-19 anos de idade, 20,5\% apresentaram sobrepeso e 4,9\% obesidade ${ }^{3}$. Em Florianópolis, Santa Catarina, a prevalência de sobrepeso/obesidade encontrada na população escolar de $7-14$ anos de idade foi de 30,3\% no ano de 2002 e 34,4\% em 2007 4,5.

Embora os fatores biológicos/genéticos sejam importantes para determinação da suscetibilidade individual ao sobrepeso/obesidade, os fatores ambientais criam o contexto para o desenvolvimento desse distúrbio ${ }^{6}$. Quando o ambiente é desfavorável em relação às práticas alimentares, ele poderá propiciar condições que levem ao desenvolvimento de sobrepeso/obesidade que, uma vez instalados, poderão permanecer ao longo da vida 7,8. Dessa forma, o ambiente no qual a criança e sua família vivem tem sido reconhecido como importante preditor das condições de saúde e nutrição 9,10,11.

A escolha/aquisição dos alimentos reflete o início da cadeia de consumo alimentar no contexto familiar, pois os alimentos que os pais compram para seus filhos e demais membros da família determinarão, em parte, a disponibilidade e a qualidade da alimentação no domicílio 12,13. Nesse sentido, sugere-se que um dos fatores vinculados ao aumento da prevalência de sobrepeso/obesidade seja a ampla disponibilidade domiciliar de alimentos industrializados/ ultraprocessados ricos em açúcar, gordura e calorias, o que estimula o consumo alimentar em quantidade excessiva e qualidade inadequada 12 .

O conhecimento dos locais utilizados pelas famílias para aquisição de alimentos e sua proximidade com os domicílios poderá fornecer subsídios para uma melhor compreensão da sua possível associação com a prevalência de sobrepeso/obesidade em escolares. Evidências indicam que existe relação entre disponibilidade e utilização dos pontos de venda de alimentos, sua proximidade com os domicílios, a prática de alimentação saudável e o sobrepeso/obesidade na população 13,14.

No presente artigo, são considerados como pontos de venda de alimentos aqueles estabelecimentos que comercializam produtos alimentícios para preparo e consumo domiciliar ou em outros ambientes diferentes do local da aquisição (supermercado, minimercado, padaria, feira/frutaria/sacolão, açougue e outros). Tais estabelecimentos apresentam como característica principal a atividade de venda de alimentos e funcionam quase sempre durante o período diurno 15 .

São escassos os estudos publicados sobre pontos de venda de alimentos e sobrepeso/obesidade em escolares realizados no Brasil. Assim, o estudo teve por objetivo verificar se existe associação entre a utilização dos pontos de venda de alimentos pelas famílias, sua proximidade com a residência e sobrepeso/obesidade em escolares de 7-14 anos de idade de escolas públicas e privadas de Florianópolis.

\section{Métodos}

Trata-se de um estudo de caráter transversal com amostra probabilística de escolares de 7-14 anos de idade matriculados no ensino fundamental de escolas públicas e privadas de Florianópolis. A justificativa da escolha dessa faixa etária teve por objetivo dar continuidade às pesquisas realizadas anteriormente com tal população (painéis transversais realizados em 2002 e 2007) 4,5. O universo do qual foi selecionada a amostra era composto de 45.247 alunos do Ensino Fundamental, distribuídos entre 85 escolas (públicas e privadas) do Município de Florianópolis que apresentavam turmas de todas as séries do Ensino Fundamental no período diurno. As informações foram obtidas do censo escolar de 2010 disponibilizado pelo Instituto Nacional de Estudos e Pesquisas Educacionais Anísio Teixeira (INEP). No cálculo do tamanho da amostra, foi considerado como desfecho o sobrepeso/obesidade em escolares, de acordo com os critérios da OMS de 2007 e adotados pelo Ministério da Saúde do Brasil, classificado como $>$ escore $-\mathrm{z}+1$ do índice de massa corporalpara-idade (IMC/idade) 16 .

De acordo com os resultados dos painéis transversais quinquenais realizados com escolares de 7-14 anos de idade na cidade de Florianópolis em 20024 e em 2007 5, que encontraram prevalências de sobrepeso/obesidade de $30 \%$ e $34 \%$, respectivamente, a prevalência esperada para 2012 seria de 38\%. Essa estimativa de aumento na prevalência de sobrepeso/obesidade foi realizada com base no encontrado nos estu- 
dos anteriores com $4 \%$ de aumento para cada quinquênio. Por se tratar de um estudo com a mesma população e desfecho, optou-se por utilizar essa prevalência para o cálculo do tamanho de amostra.

Considerando um erro amostral de 3,5 pontos percentuais (bicaudal) e um intervalo de $95 \%$ de confiança (IC95\%); o tamanho de amostra necessário para a pesquisa seria de 727 escolares. Utilizando um efeito de delineamento (DEFF) de 1.8 (estimado com base na pesquisa de 2007) 5 , o tamanho de amostra total necessário seria 1.309 escolares. Ponderando a possibilidade de realizar comparações com as informações coletadas em 2007, quando os dados foram estratificados por faixa etária (7-10 anos e 11-14 anos), esse tamanho de amostra foi duplicado. Acrescentando $10 \%$ a esse valor por eventuais perdas ou recusas à pesquisa, o tamanho de amostra final foi de 2.880 escolares.

O processo de amostragem foi realizado por conglomerados, tendo como unidades amostrais primárias as 85 escolas do município que apresentavam turmas de todas as séries do Ensino Fundamental no período diurno. Tendo por objetivo garantir a variabilidade amostral, para a seleção das escolas, elas foram inicialmente divididas em 10 estratos, de acordo com as regiões administrativas do Município de Florianópolis (Centro, Continente, Norte, Leste e Sul) e tipo de escola (pública ou privada). Em cada estrato, foram selecionadas aleatoriamente as escolas a serem incluídas no estudo. Posteriormente, realizou-se a seleção das turmas em cada escola, por meio de um processo de amostragem sistemática com base na lista de escolares disponibilizada em cada escola. Considerando os dados da pesquisa de 20075 e o número total de escolas disponíveis para essa pesquisa $(n=85)$, foi selecionado um total de 30 escolas, sendo 19 públicas e 11 privadas (visando à garantia da variabilidade socioeconômica da amostra avaliada), correspondendo a $35 \%$ do total, e em cada uma foram avaliados em média 100 escolares.

Esclarece-se que considerando o tamanho de amostra disponível, a prevalência do desfecho e das diversas variáveis independentes avaliadas, o presente estudo teve $80 \%$ de poder para detectar razão de prevalência (RP) entre 1,36 e 1,40 como fatores de risco, bem como valores entre $0,72 \mathrm{e}$ 0,74 como fatores de proteção. Tais estimativas consideraram $5 \%$ de nível alfa, $10 \%$ de perdas e ajuste de $15 \%$ para fatores de confusão, levando em consideração o efeito de delineamento $(1,8)$.

Participaram da pesquisa aqueles escolares que apresentaram a permissão dos pais ou responsáveis pela assinatura de Termo de Consentimento Livre e Esclarecido (TCLE). Foram excluídos do estudo escolares portadores de deficiência física que impossibilitasse a avaliação antropométrica e adolescentes grávidas. Como perdas ou recusas, foram levados em conta aqueles escolares que não retornaram o TCLE dos pais assinado, aqueles com dados incompletos sobre o perfil de aquisição de alimentos, ou ainda aqueles que mesmo com o consentimento dos pais recusaram participar do estudo.

A equipe responsável pela coleta dos dados foi treinada em dois momentos, por meio de seminários de padronização de medidas antropométricas e aplicação de demais instrumentos da coleta de dados, com base em atividades teóricas e práticas relativas à técnica de mensuração. De acordo com o protocolo de Habicht 17, foi realizado treinamento para a padronização entre examinadores e estudo de variabilidade intra e interexaminadores. Antecedendo a coleta de dados, foi realizado estudo piloto que teve por objetivo uniformizar os procedimentos de coleta e averiguar a aplicabilidade dos instrumentos propostos.

As tomadas das medidas antropométricas foram realizadas segundo protocolos estabelecidos na literatura ${ }^{18}$. A medida da massa corporal foi obtida utilizando uma balança eletrônica da marca Marte, modelo LC 200 PP (Marte Científica, São Paulo, Brasil), com capacidade mínima de $1 \mathrm{~kg}$ e máxima de $199,95 \mathrm{~kg}$ e precisão de 50 gramas. Para obtenção da medida da estatura, foi utilizado um estadiômetro da marca Alturexata, com precisão de um milímetro (Alturexata, Belo Horizonte, Brasil). O procedimento foi realizado com os escolares vestindo roupas leves, descalços, na posição ortostática (em pé e corpo ereto), com a massa corporal dividida em ambos os membros inferiores, braços soltos lateralmente ao corpo, ombros descontraídos e mantendo a cabeça no plano de Frankfurt (plano para a orientação cefálica, no qual o indivíduo permanece com o olhar no horizonte, e uma linha imaginária passa na cabeça, tangendo a borda superior dos condutos auditivos externos e o ponto mais baixo na margem da órbita ocular). Na medida de estatura, foi solicitado também que o avaliado respirasse fundo. Essa técnica tem como objetivo diminuir o efeito da compressão gravitacional diária.

No questionário enviado juntamente com TCLE aos pais ou responsáveis dos escolares, constaram informações socioeconômicas e demográficas como sexo e idade do escolar, identificação do chefe da família, escolaridade paterna e materna, renda familiar e tipo de unidade administrativa de ensino em que o escolar estava inserido. Essas variáveis foram categorizadas como variáveis de ajuste por causa de sua associa- 
ção com o desfecho. Por sua vez, também foram obtidas informações sobre o perfil de aquisição de alimentos (principais variáveis de exposição) por perguntas relacionadas a: quais os tipos de pontos de venda de alimentos utilizados pelas famílias dos escolares e o tempo de deslocamento a pé da residência da família até esses locais. A última variável teve por objetivo estimar a proximidade dos pontos de vendas de alimentos utilizados até a residência da família. Essas perguntas foram feitas na modalidade fechada com múltipla escolha.

Para as análises, foram consideradas variáveis independentes: sexo (masculino e feminino), idade (7-10 anos e 11-14 anos), chefe da família (mãe, pai, outros), escolaridade paterna e materna (sem instrução/Ensino Fundamental completo; Ensino Fundamental completo/Ensino Médio incompleto; Ensino Médio completo/ Ensino Superior incompleto e Ensino Superior completo), renda mensal familiar (coletada em reais e categorizada em 1o, 2o e 3 o tercil de distribuição), tipo de unidade administrativa de ensino frequentada pelo escolar (pública ou privada), tipos de pontos de venda de alimentos utilizados pelas famílias (supermercado, minimercado, feira/frutaria/sacolão, padaria, açougue, outros) e tempo de deslocamento a pé da residência da família até os estabelecimentos (até 10 minutos, 11-20 minutos e mais de 20 minutos). A variável dependente considerada foi o sobrepeso/obesidade do escolar segundo o IMC, utilizando a classificação proposta pela OMS 16. Essa variável foi categorizada em presença de sobrepeso ou obesidade (IMC > escore- $\mathrm{Z}+1$ ) e ausência de sobrepeso ou obesidade (IMC $\leq$ escore- $\mathrm{Z}+1$ ).

Em relação à variável tempo de deslocamento/proximidade até os pontos de venda de alimentos, entendeu-se que em média um adulto pode percorrer 400 metros em 5 minutos $19 \mathrm{e}$ adolescentes, em ritmo moderado, são capazes de percorrer 1.600 metros em 15 minutos 20. De posse dessas informações, foi possível fazer uma inferência da distância entre os pontos de venda de alimentos utilizados pela família, considerando que um adulto poderia percorrer em $10 \mathrm{mi}$ nutos até 800 metros, de 11 a 20 minutos seria possível percorrer uma distância a pé entre $800 \mathrm{~m}$ e 1.600 metros; em deslocamentos superiores a 20 minutos, a distância percorrida corresponderia a mais de $1,6 \mathrm{~km}$.

Para a análise estatística, foi utilizado o software Stata versão 11.0 (Stata Corp., College Station, Estados Unidos). A análise dos dados foi feita de forma descritiva e inferencial, por intermédio de testes estatísticos para analisar possíveis associações entre a prevalência de sobrepeso/ obesidade nos escolares, a utilização do pontos de venda de alimentos e sua proximidade com a residência da família. Como procedimento de ajuste foi utilizado o comando svy para considerar o efeito do delineamento e plano amostral nas análises. Para analisar a associação entre a variável dependente e cada variável independente, foi realizada análise bivariada. Todas as variáveis que apresentaram valor de $\mathrm{p}<0,20$ foram incluídas na análise multivariada, utilizando o método de regressão de Poisson. O critério de permanência no modelo final foi valor de $\mathrm{p} \leq$ 0,05 . Os resultados são apresentados pelas razões de prevalências e respectivos IC95\%.

O protocolo da pesquisa foi aprovado pelo Comitê de Ética da Pesquisa com Seres Humanos da Universidade Federal de Santa Catarina (parecer no 120.341/2012), de acordo com as normas estabelecidas pela Resolução no 466/2012 do Conselho Nacional de Saúde 21.

\section{Resultados}

Um total de 4.082 escolares nas 30 escolas participantes da pesquisa, com idade de 7-14 anos, foram considerados elegíveis para o estudo. Desses, apenas 2.506 retornaram o TCLE devidamente assinado pelos pais (61,4\% dos elegíveis), sendo 1.637 escolares da rede pública (65,3\%) e 869 da rede privada $(34,7 \%)$.

A caracterização geral dos participantes é apresentada na Tabela 1, de acordo com o tipo de escola. Foram avaliados mais escolares na faixa etária de $7-10$ anos $(62,4 \%$ na rede pública e $61,6 \%$ na rede privada). A prevalência de sobrepeso e obesidade foi de $34,2 \%$ na amostra, sem diferença estatisticamente significativa entre escolares da rede pública e privada. Entre os escolares da rede pública, $19,6 \%$ apresentaram sobrepeso e $13,5 \%$ obesidade, $22,4 \%$ dos escolares da rede privada estavam com sobrepeso e 11,1\% com obesidade. Entretanto, diferenças estatisticamente significativas foram encontradas entre escolares da rede pública e privada de ensino quando consideradas as variáveis chefe da família, escolaridade paterna e materna e renda mensal familiar.

Na Tabela 2, estão descritas as características da amostra de acordo com a utilização dos pontos de venda de alimentos e sua proximidade até a residência da família, estratificada por tipo de escola. Constatou-se que, para famílias de escolares da rede pública, o local habitual de aquisição de alimentos foi o supermercado $(96,6 \%)$, seguido por feira/frutaria/sacolão $(90,4 \%)$, padaria (88,9\%), minimercado $(69,3 \%)$, açougue $(52,4 \%)$ e outros locais como peixarias e casa de produtos naturais $(17,3 \%)$. Foram encontrados resultados 
Distribuição das características socioeconômicas e demográficas da amostra estratificada por tipo de escola. Florianópolis, Santa Catarina, Brasil, 2012/2013.

\begin{tabular}{|c|c|c|c|c|c|c|c|}
\hline \multirow[t]{2}{*}{ Variável } & \multicolumn{2}{|c|}{ Total } & \multicolumn{2}{|c|}{ Pública } & \multicolumn{2}{|c|}{ Privada } & \multirow{2}{*}{$\begin{array}{l}\text { Valor } \\
\text { de } p \text { * }\end{array}$} \\
\hline & n & $\%$ & $\mathbf{n}$ & $\%$ & $\mathbf{n}$ & $\%$ & \\
\hline Idade do escolar (anos) & & & & & & & 0,846 \\
\hline $7-10$ & 1.531 & 62,0 & 1.002 & 62,4 & 529 & 61,6 & \\
\hline $11-14$ & 975 & 38,0 & 635 & 37,6 & 340 & 38,4 & \\
\hline Sexo & & & & & & & 0,961 \\
\hline Masculino & 1.172 & 46,6 & 764 & 46,7 & 408 & 46,6 & \\
\hline Feminino & 1.334 & 53,4 & 873 & 53,3 & 461 & 53,4 & \\
\hline Chefe da família ** & & & & & & & $<0,001$ \\
\hline Mãe & 910 & 36,7 & 629 & 40,5 & 281 & 33,5 & \\
\hline Pai & 1.343 & 57,3 & 819 & 51,9 & 524 & 61,9 & \\
\hline Outros & 150 & 6,0 & 112 & 7,6 & 38 & 4,6 & \\
\hline Escolaridade materna ** & & & & & & & $<0,001$ \\
\hline Sem instrução e Ensino Fundamental incompleto & 469 & 13,2 & 461 & 27,7 & 8 & 9,2 & \\
\hline Ensino Fundamental completo e Ensino Médio incompleto & 382 & 12,5 & 347 & 22,3 & 35 & 4,2 & \\
\hline Ensino Médio completo e Ensino Superior incompleto & 857 & 35,5 & 581 & 35,0 & 276 & 32,1 & \\
\hline Ensino Superior completo & 681 & 38,8 & 147 & 10,5 & 534 & 62,8 & \\
\hline Escolaridade paterna ** & & & & & & & $<0,001$ \\
\hline Sem instrução e Ensino Fundamental incompleto & 447 & 14,7 & 429 & 30,4 & 18 & 2,4 & \\
\hline Ensino Fundamental completo e Ensino Médio incompleto & 359 & 14,1 & 313 & 24,7 & 46 & 5,8 & \\
\hline Ensino Médio completo e Ensino Superior incompleto & 710 & 34,7 & 435 & 35,1 & 275 & 34,4 & \\
\hline Ensino Superior completo & 570 & 36,6 & 126 & 9,8 & 444 & 57,5 & \\
\hline Renda familiar ** & & & & & & & $<0,001$ \\
\hline 1ㅇ tercil (R\$ 136,00 a $R \$ 1.576,00)$ & 718 & 25,8 & 672 & 44,6 & 46 & 6,8 & \\
\hline 2o tercil $(\mathrm{R} \$ 1.577,00$ a $\mathrm{R} \$ 3.000,00)$ & 736 & 31,2 & 585 & 40,9 & 151 & 21,5 & \\
\hline 3o tercil ( $R \$ 3.001,00$ a $R \$ 80.000,00)$ & 698 & 43,0 & 201 & 14,4 & 497 & 71,7 & \\
\hline Estado nutricional dos escolares *** & & & & & & & 0,925 \\
\hline Sem sobrepeso/obesidade & 1.658 & 65,8 & 1084 & 65,6 & 574 & 66,0 & \\
\hline Com sobrepeso/obesidade & 826 & 34,2 & 537 & 34,4 & 289 & 34,0 & \\
\hline Total & 2.506 & 100,0 & 1.637 & 65,3 & 869 & 34,7 & \\
\hline
\end{tabular}

* Teste qui-quadrado;

** Variável com dados ignorados;

*** Valores categorizados de acordo com a Organização Mundial da Saúde.

similares em famílias de escolares da rede privada de ensino, para as quais o ponto de venda de alimentos preferencial de utilização foi o supermercado $(96,2 \%)$, seguido por feira/frutaria/ sacolão $(87,5 \%)$, padaria $(87,8 \%)$, minimercado $(64,0 \%)$, açougue $(43,8 \%)$ e outros locais como peixarias e casa de produtos naturais $(21,6 \%)$. Nenhuma dessas variáveis apresentou diferença estatisticamente significativa entre escolares da rede pública e privada de ensino.

Em geral, o tempo de deslocamento da residência da família até os pontos de venda de alimentos entre os escolares da rede pública $\mathrm{e}$ privada apresentou-se distribuído da seguin- te maneira: a maior frequência foi o tempo de deslocamento de até 10 minutos e o de menor frequência foi o tempo de deslocamento superior a 20 minutos. A única exceção foi o tempo de deslocamento da residência até o supermercado em famílias de escolares da rede privada, prevalecendo o tempo de deslocamento de 11-20 minutos, seguido por tempo de deslocamento superior a 20 minutos e, por fim, o tempo de deslocamento a pé em até 10 minutos.

$\mathrm{Na}$ análise bruta, foi encontrada associação entre uso de padaria e sobrepeso/obesidade nos escolares da rede pública de ensino $(\mathrm{p}=$ $0,015)$. Essa associação permaneceu na ajustada 
Distribuição das características da amostra de acordo com a utilização do ponto de venda de alimentos e tempo de deslocamento até a residência estratificada por tipo de escola. Florianópolis, Santa Catarina, Brasil, $2012 / 2013$.

\begin{tabular}{|c|c|c|c|c|c|c|c|}
\hline \multirow[t]{2}{*}{ Variável } & \multicolumn{2}{|c|}{ Total } & \multicolumn{2}{|c|}{ Pública } & \multicolumn{2}{|c|}{ Privada } & \multirow[t]{2}{*}{ Valor de $p$ * } \\
\hline & $\mathbf{n}$ & $\%$ & $\mathrm{n}$ & $\%$ & $\mathrm{n}$ & $\%$ & \\
\hline $\begin{array}{l}\text { Ponto de venda de alimentos utilizado e tempo de } \\
\text { deslocamento (minutos) }\end{array}$ & & & & & & & 0,466 \\
\hline Supermercado & 2.306 & 96,4 & 1.460 & 96,6 & 815 & 96,2 & \\
\hline Até 10 & 894 & 38,8 & 559 & 38,4 & 230 & 28,2 & \\
\hline $11-20$ & 662 & 28,7 & 432 & 30,6 & 335 & 41,1 & \\
\hline Mais de 20 & 700 & 30,3 & 469 & 31,0 & 231 & 28,3 & \\
\hline Não responderam & 50 & 2,2 & & & 19 & 2,4 & \\
\hline Minimercado & 1.451 & 66,5 & 961 & 69,3 & 490 & 64,0 & 0,388 \\
\hline Até 10 & 1.048 & 72,2 & 695 & 72,3 & 353 & 72,6 & \\
\hline $11-20$ & 261 & 17,9 & 176 & 18,3 & 85 & 16,9 & \\
\hline Mais de 20 & 133 & 9,1 & 81 & 8,4 & 52 & 10,5 & \\
\hline Não responderam & 9 & 0,8 & 9 & 1,0 & & & \\
\hline Feira/Frutaria/Sacolão & 2.066 & 88,8 & 1.343 & 90,4 & 723 & 87,5 & 0,879 \\
\hline Até 10 & 829 & 40,1 & 551 & 41,0 & 278 & 38,4 & \\
\hline $11-20$ & 662 & 32,0 & 427 & 31,7 & 235 & 32,5 & \\
\hline Mais de 20 & 515 & 24,9 & 324 & 24,1 & 191 & 26,4 & \\
\hline Não responderam & 60 & 3,0 & 41 & 3,2 & 19 & 2,7 & \\
\hline Padaria & 2.000 & 88,3 & 1.283 & 88,9 & 717 & 87,8 & 0,658 \\
\hline Até 10 & 1.305 & 65,3 & 835 & 65,0 & 470 & 65,5 & \\
\hline $11-20$ & 437 & 21,8 & 287 & 22,3 & 150 & 20,9 & \\
\hline Mais de 20 & 204 & 10,2 & 128 & 9,9 & 76 & 10,5 & \\
\hline Não responderam & 54 & 2,7 & 33 & 2,8 & 21 & 3,1 & \\
\hline Açougue & 995 & 47,7 & 672 & 52,4 & 323 & 43,8 & 0,263 \\
\hline Até 10 & 464 & 46,6 & 307 & 45,6 & 157 & 48,6 & \\
\hline $11-20$ & 277 & 27,8 & 193 & 28,7 & 84 & 26,0 & \\
\hline Mais de 20 & 237 & 23,8 & 156 & 23,2 & 81 & 25,0 & \\
\hline Não responderam & 17 & 1,8 & 16 & 2,5 & 1 & 0,4 & \\
\hline Outros & 168 & 19,8 & 90 & 17,3 & 78 & 21,6 & 0,106 \\
\hline Até 10 & 57 & 33,9 & 30 & 33,3 & 27 & 34,6 & \\
\hline $11-20$ & 29 & 17,2 & 10 & 11,1 & 19 & 24,3 & \\
\hline Mais de 20 & 47 & 27,9 & 29 & 32,2 & 18 & 23,0 & \\
\hline Não responderam & 35 & 21,0 & 21 & 23,4 & 14 & 18,1 & \\
\hline
\end{tabular}

* Teste qui-quadrado.

realizada pela regressão de Poisson $(\mathrm{p}=0,004)$ Entre os escolares da rede privada de ensino, foi constatada a associação entre a utilização de supermercado e sobrepeso/obesidade ( $\mathrm{p}=$ 0,003) (Tabela 3).

\section{Discussão}

Na presente investigação, a prevalência de sobrepeso/obesidade encontrada na amostra foi de $34,2 \%$, sem diferença estatisticamente signi- ficativa entre escolares da rede pública e privada. Ao comparar essa prevalência com estudos internacionais, que utilizaram o mesmo critério de diagnóstico e faixa etária similar, prevalências mais aproximadas foram obtidas na Itália (35\%) 22. Maior prevalência foi verificada entre crianças e adolescentes americanos (47\%) na faixa etária de 6-19 anos 2 . Apesar de ainda serem encontradas altas prevalências de sobrepeso/obesidade em pré-escolares e escolares no contexto mundial, nas duas últimas décadas, observa-se uma estabilização nas prevalências 
Tabela 3

Análise bruta e ajustada da prevalência de sobrepeso/obesidade e utilização do ponto de venda de alimentos estratificado por tipo de escola. Florianópolis, Santa Catarina, Brasil, 2012/2013.

\begin{tabular}{|c|c|c|c|c|c|c|c|c|}
\hline \multirow[t]{3}{*}{ Variáveis } & \multicolumn{8}{|c|}{ Prevalência de sobrepeso/obesidade } \\
\hline & \multicolumn{4}{|c|}{ Escola pública } & \multicolumn{4}{|c|}{ Escola privada } \\
\hline & $\begin{array}{l}\text { RP bruta } \\
\text { (IC95\%) }\end{array}$ & Valor de p * & $\begin{array}{l}\text { RP ajustada } \\
\text { (IC95\%) ** }\end{array}$ & $\begin{array}{l}\text { Valor de } \\
p^{\star \star \star}\end{array}$ & $\begin{array}{l}\text { RP bruta } \\
\text { (IC95\%) }\end{array}$ & Valor de p * & $\begin{array}{l}\text { RP ajustada } \\
\text { (IC95\%) ** }\end{array}$ & $\begin{array}{l}\text { Valor de } \\
p^{\star \star \star}\end{array}$ \\
\hline \multicolumn{9}{|c|}{$\begin{array}{l}\text { Pontos de venda de alimentos } \\
\text { utilizado pela família }\end{array}$} \\
\hline \multicolumn{9}{|c|}{ Supermercado } \\
\hline Não & 1,00 & 0,815 & 1,00 & 0,550 & 1,00 & 0,017 & 1,00 & 0,003 \\
\hline Sim & $0,95(0,61-1,47)$ & & $0,86(0,53-1,41)$ & & $0,79(0,45-0,94)$ & & $0,74(0,41-0,97)$ & \\
\hline \multicolumn{9}{|c|}{ Minimercado } \\
\hline Não & 1,00 & 0,399 & 1,00 & 0,436 & 1,00 & 0,427 & 1,00 & 0,497 \\
\hline Sim & $0,92(0,76-1,12)$ & & $0,91(0,73-1,15)$ & & $0,90(0,70-1,16)$ & & $0,90(0,66-1,22)$ & \\
\hline \multicolumn{9}{|c|}{ Feira/Frutaria/Sacolão } \\
\hline Não & 1,00 & 0,213 & 1,00 & 0,349 & 1,00 & 0,391 & 1,00 & 0,390 \\
\hline Sim & $1,22(0,89-1,67)$ & & $1,19(0,83-1,72)$ & & $1,19(0,80-1,76)$ & & $1,22(0,78-1,91)$ & \\
\hline \multicolumn{9}{|l|}{ Padaria } \\
\hline Não & 1,00 & 0,015 & 1,00 & 0,004 & 1,00 & 0,687 & 1,00 & 0,947 \\
\hline Sim & $1,09(1,03-1,43)$ & & $1,18(1,08-1,78)$ & & $1,08(0,74-1,57)$ & & $1,01(0,66-1,56)$ & \\
\hline \multicolumn{9}{|c|}{ Açougue } \\
\hline Não & 1,00 & 0,795 & 1,00 & 0,954 & 1,00 & 0,559 & 1,00 & 0,346 \\
\hline Sim & $0,98(0,81-1,18)$ & & $0,99(0,80-1,24)$ & & $0,93(0,71-1,20)$ & & $0,86(0,63-1,18)$ & \\
\hline \multicolumn{9}{|l|}{ Outros } \\
\hline Não & 1,00 & 0,569 & 1,00 & 0,304 & 1,00 & 0,306 & 1,00 & 0,210 \\
\hline Sim & $1,12(0,76-1,66)$ & & $1,26(0,81-1,94)$ & & $0,78(0,48-1,26)$ & & $0,71(0,41-1,22)$ & \\
\hline
\end{tabular}

IC95\%: intervalo de 95\% de confiança; RP: razão de prevalência.

* Teste qui-quadrado;

** Razão de prevalência ajustada para sexo, idade, chefe da família, escolaridade paterna e materna e renda mensal familiar;

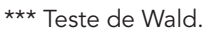

dessa doença em alguns países como Dinamarca e Groenlândia 23.

Esses achados corroboram com as pesquisas nacionais em relação ao aumento do sobrepeso/obesidade nessa população 4,5 . A prevalência identificada nesta pesquisa é preocupante, pois os escolares também são afetados pelas consequências do sobrepeso/obesidade por conta de sua forte relação com as DCNT 1,2.

O supermercado foi o estabelecimento mais citado pelos participantes como o principal ponto de venda de alimentos utilizado por famílias dos escolares da amostra, similarmente ao encontrado em estudo realizado sobre os hábitos de compra da população de Ouro Preto, Minas Gerais 24. Os supermercados, caracterizados como grandes lojas de varejo, possuem grande diversidade de alimentos considerados saudáveis com preços competitivos e de qualidade superior quando comparados aos outros pontos de venda de ali- mentos 25,26, atributos reconhecidos como pontos positivos de tais estabelecimentos. Porém, é preciso ressaltar que esses estabelecimentos também são grandes fornecedores de ampla diversidade de alimentos processados com alto teor de gordura, açúcar e calorias que podem contribuir para o aumento do sobrepeso/obesidade da população 27 .

Existem na literatura evidências sobre a associação entre a disponibilidade e proximidade dos pontos de venda de alimentos até a residência da família e estado nutricional da população 6,28. Esses estudos sobre as relações entre a disponibilidade de supermercado e alimentação sugerem que o fato de existir um supermercado perto das residências facilita a aquisição de alimentos saudáveis (vegetais frescos, por exemplo) e podem diminuir a predisposição ao sobrepeso/ obesidade 28,29 . 
Nesta pesquisa, foi observada entre os escolares da rede privada associação positiva e estatisticamente significativa entre a menor prevalência de sobrepeso/obesidade e a utilização de supermercado. Resultados semelhantes foram encontrados na China, em estudo envolvendo 185 estudantes com idade de 6-18 anos, que tinha por objetivo investigar se a disponibilidade de grandes supermercados no entorno das residências das famílias estava relacionada com o sobrepeso/obesidade dos estudantes. Os resultados encontrados mostraram que com menores preços e oferta de alimentos mais frescos, os supermercados são mais suscetíveis a contribuir com uma alimentação menos calórica e melhorar o estado nutricional da população 30 . Sendo assim, a utilização de supermercados pelas famílias pode ser justificada pela praticidade oferecida por esses estabelecimentos comerciais como a possibilidade da utilização de cartão de crédito e tíquete-alimentação para a realização das compras, além dos serviços de entrega no domicílio.

$\mathrm{O}$ achado na presente pesquisa é semelhante ao observado em estudo longitudinal com meninas de sete anos de idade acompanhadas durante três anos na Califórnia, Estados Unidos. Ao analisar a relação entre a disponibilidade de pontos de venda de alimentos no bairro e risco de sobrepeso/obesidade ao longo da pesquisa, verificou-se uma tendência significativa entre a maior disponibilidade de supermercados ao longo do estudo e menor risco de sobrepeso/obesidade após três anos de acompanhamento 31 .

Nos escolares da rede pública, constattou-se associação positiva e estatisticamente significativa entre utilização de padaria e prevalência de sobrepeso/obesidade. A padaria pode ser definida como pequeno estabelecimento de comércio varejista, localizado nos bairros, que comercializa basicamente produtos panificados, laticínios, doces, balas e com pouca oferta de vegetais 32,33 .

Estudo realizado em Nova Iorque (Estados Unidos) com 323 escolares de 6 a 8 anos de idade encontrou que a presença de padarias perto da residência foi associada a um maior IMC dentre a população. Resultados semelhantes foram encontrados em outro estudo realizado nos Estados Unidos, onde o acesso a padarias foi associado ao maior sobrepeso e obesidade entre os adultos 14 .

Fatores econômicos parecem exercer influência decisiva na ingestão de alimentos saudáveis (frutas e hortaliças, por exemplo). Evidências indicam que dietas com alto teor de vegetais são mais caras do que as demais, e que a imposição de restrições econômicas ao custo da alimentação (como aquelas vivenciadas por famílias economicamente menos favorecidas) conduz a die- tas com baixa participação de frutas e hortaliças e maior consumo de alimentos de alta densidade energética (especialmente pelo alto teor de cereais processados, óleo e açúcar) 34,35.

Os resultados encontrados no presente estudo corroboram com essas evidências; em famílias de escolares da rede privada, com melhores condições de renda e possivelmente com mais facilidade para o deslocamento até o supermercado, foi verificada uma menor prevalência de sobrepeso/obesidade quando comparadas às famílias que não utilizaram esses pontos de venda de alimentos. Entre famílias de escolares da rede pública, com provável menor poder aquisitivo e que utilizam com maior frequência a padaria para aquisição de alimentos, foi verificada uma maior prevalência de sobrepeso/obesidade na população avaliada. A presença de padarias no bairro pode ser um fator de risco para o desenvolvimento de sobrepeso/obesidade em escolares, pois tais pontos de venda de alimentos funcionam muitas horas ao dia (o que facilita o acesso pelos consumidores locais), possuem grande disponibilidade de alimentos panificados, fonte de carboidratos e calorias, e pouca oferta de vegetais frescos para aquisição 36 .

No presente estudo, não foi verificada associação estatisticamente significativa entre a utilização de feira/frutaria/sacolão e presença de sobrepeso/obesidade na população avaliada. Contudo, em estudo realizado em 52 setores cencitários do Município de São Paulo foi encontrada associação significativa entre consumo de frutas e a disponibilidade de frutarias próximas aos domicílios de adultos que residem em regiões de nível socioeconômico médio e alto. Essa evidência mostra que o acesso aos locais que comercializam alimentos saudáveis deve ser considerado nos estudos sobre ambiente alimentar e sobrepeso/obesdiade da população ${ }^{37}$.

Os resultados mostram que não houve associação estatisticamente significativa entre a prevalência de sobrepeso/obesidade e a proximidade da residência até os pontos de venda de alimentos utilizados pela família (Tabela 4). Alguns estudos que procuraram relacionar a disponibilidade dos tipos de pontos de venda de alimentos, o tempo de deslocamento deles até as residências e o consumo dos alimentos citam que disponibilidade, preço e qualidade são fortes influentes das decisões de compra de alimentos e decisivos nos padrões alimentares da população 38,39. Na mesma linha, estudo realizado com crianças em Massachusetts (Estados Unidos), que teve por objetivo analisar a associação entre a proximidade de pontos de venda de alimentos com o IMC de crianças entre 2-9 anos de idade, encontrou que residir perto de um grande supermercado 
Tabela 4

Análise bruta e ajustada da prevalência de sobrepeso/obesidade e tempo de deslocamento a pé da residência até o ponto de venda de alimentos estratificado por tipo de escola. Florianópolis, Santa Catarina, Brasil, 2012/2013.

\begin{tabular}{|c|c|c|c|c|c|c|c|c|}
\hline \multirow[t]{3}{*}{ Variáveis } & \multicolumn{8}{|c|}{ Prevalência de sobrepeso/obesidade } \\
\hline & \multicolumn{4}{|c|}{ Escola pública } & \multicolumn{4}{|c|}{ Escola privada } \\
\hline & $\begin{array}{l}\text { RP bruta } \\
\text { (IC95\%) }\end{array}$ & $\begin{array}{l}\text { Valor } \\
\text { de } p\end{array}$ & $\begin{array}{l}\text { RP ajustada } \\
\text { (IC95\%) * }\end{array}$ & $\begin{array}{l}\text { Valor } \\
\text { de } p\end{array}$ & $\begin{array}{l}\text { RP bruta } \\
\text { (IC95\%) }\end{array}$ & $\begin{array}{l}\text { Valor } \\
\text { de } p\end{array}$ & $\begin{array}{l}\text { RP ajustada } \\
\text { (IC95\%) * }\end{array}$ & Valor de $p$ \\
\hline \multicolumn{9}{|c|}{$\begin{array}{l}\text { Tempo de deslocamento da } \\
\text { residência até o ponto de } \\
\text { venda de alimentos (minutos) }\end{array}$} \\
\hline \multicolumn{9}{|l|}{ Supermercado } \\
\hline Até 10 & 1,00 & 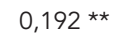 & 1,00 & 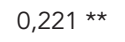 & 1,00 & 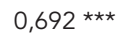 & 1,00 & $0,575^{\star \star}$ \\
\hline $11-20$ & $1,02(0,1-1,28)$ & & $1,08(0,83-1,41)$ & & $0,97(0,72-1,29)$ & & $0,96(0,68-1,35)$ & \\
\hline Mais de 20 & $1,20(0,97-1,48)$ & & $1,24(0,97-1,59)$ & & $0,88(0,65-1,18)$ & & $0,83(0,59-1,18)$ & \\
\hline \multicolumn{9}{|l|}{ Minimercado } \\
\hline Até 10 & 1,00 & 0,537 ** & 1,00 & 0,284 ** & 1,00 & 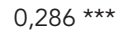 & 1,00 & 0,288 ** \\
\hline $11-20$ & $1,07(0,80-1,43)$ & & $1,21(0,85-1,71)$ & & $0,83(0,53-1,29)$ & & $0,72(0,41-1,26)$ & \\
\hline Mais de 20 & $1,23(0,85-1,80)$ & & $1,34(0,87-2,07)$ & & $0,64(0,34-1,18)$ & & $0,65(0,33-1,30)$ & \\
\hline \multicolumn{9}{|c|}{ Feira/Frutaria/Sacolão } \\
\hline Até 10 & 1,00 & 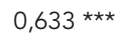 & 1,00 & 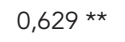 & 1,00 & 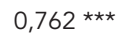 & 1,00 & 0,405 *夫 \\
\hline $11-20$ & $1,08(0,87-1,34)$ & & $1,09(0,85-1,40)$ & & $0,90(0,65-1,23)$ & & $0,87(0,61-1,24)$ & \\
\hline Mais de 20 & $0,96(0,75-1,22)$ & & $0,95(0,71-1,26)$ & & $0,36(0,29-0,43)$ & & $0,77(0,53-1,13)$ & \\
\hline \multicolumn{9}{|l|}{ Padaria } \\
\hline Até 10 & 1,00 & 0,709 ** & 1,00 & 0,412 ** & 1,00 & $0,110 * \star$ & 1,00 & $0,270 * \star \star$ \\
\hline $11-20$ & $1,01(0,79-1,27)$ & & $1,09(0,83-1,42)$ & & $1,44(1,00-2,08)$ & & $0,95(0,64-1,41)$ & \\
\hline Mais de 20 & $1,14(0,83-1,55)$ & & $1,26(0,89-1,80)$ & & $0,32(0,27-0,38)$ & & $1,39(0,91-2,13)$ & \\
\hline \multicolumn{9}{|l|}{ Açougue } \\
\hline Até 10 & 1,00 & 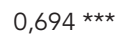 & 1,00 & $0,690 * \star \star$ & 1,00 & $0,506 * \star \star$ & 1,00 & $0,176 * \star \star$ \\
\hline $11-20$ & $1,06(0,77-1,45)$ & & $1,14(0,79-1,64)$ & & $1,01(0,63-1,61)$ & & $0,56(0,28-1,11)$ & \\
\hline Mais de 20 & $0,89(0,62-1,28)$ & & $0,96(0,63-1,46)$ & & $0,34(0,26-0,44)$ & & $0,69(0,39-1,23)$ & \\
\hline \multicolumn{9}{|l|}{ Outros } \\
\hline Até 10 & 1,00 & $0,677 \star \star \star$ & 1,00 & $0,991 * \star \star$ & 1,00 & $0,991 \star \star$ & 1,00 & 0,828 ** \\
\hline $11-20$ & $0,83(0,23-3,08)$ & & $1,14(0,15-8,52)$ & & $0,94(0,31-2,87)$ & & $1,68(0,30-9,32)$ & \\
\hline Mais de 20 & $1,34(0,57-3,13)$ & & $0,98(0,33-2,88)$ & & $0,30(0,15-0,59)$ & & $1,37(0,33-5,71)$ & \\
\hline
\end{tabular}

IC95\%: intervalo de 95\% de confiança; RP: razão de prevalência.

* Razão de prevalência ajustada para sexo, idade, chefe da família, escolaridade paterna e materna e renda mensal familiar;

** Teste de Wald de tendência;

*** Teste de Wald de heterogeneidade.

(distâncias de 300, 800 ou 1.600 metros), foi associado com menor IMC nessa população 40 . Vale ressaltar que o fato de o presente estudo não ter encontrado associação entre o tempo de deslocamento da residência até os pontos de venda de alimentos utilizados pela família e a prevelência de sobrepeso/obesidade em escolares pode ser em razão da utilização de diferentes meios de tranporte pelas famílias, e a dificuldade por parte delas em estimar o deslocamento a pé.

Estabelecida a associação do ambiente com o sobrepeso/obesidade em escolares 9,10,11, torna- se necessário abordar o ambiente físico e social no planejamento de intervenções na comunidade para reduzir os problemas de comportamento alimentar e nutricional das crianças e adolescentes 41 . Nesse contexto, sugere-se que estratégias de intervenção, de acordo com as características de cada estabelecimento, que incluam aumento da oferta de alimentos mais saudáveis em alguns tipos de pontos de venda de alimentos, além de disponibilização de informações nutricionais nas etiquetas das prateleiras e pôsteres sobre os produtos comercializados, sejam realizadas em 
pequenos pontos de venda de alimentos (minimercados, feiras, padarias e açougues). Essas iniciativas poderão incentivar o consumo de alimentos considerados saudáveis como alimentos integrais, frutas, legumes e verduras ricos em vitaminas, minerais e fibras e com baixo teor de gorduras e sal, propiciando escolhas alimentares mais saudáveis e, consequentemente, menores riscos de desenvolvimento de DCNT, com destaque para o sobrepeso/obesidade na população escolar. Ações similares já foram desenvolvidas em outros estudos com resultados significativamente positivos $42,43,44$.

Uma possível limitação é inerente ao desenho transversal do estudo, com exposição e desfecho coletados simultaneamente, sem a possibilidade de avaliar a causalidade. Além disso, o questionário contendo informações sobre a utilização dos pontos de venda de alimentos foi autopreenchido pelos pais ou responsáveis, o que pode acarretar perdas de informação. Tentando diminuir essa limitação, foi realizado um controle de qualidade dos questionários e complementação dos mesmos mediante ligações telefônicas, quando necessário. Adicionalmente, é preciso mencionar que o percentual de recusas (não adesão) foi alto (38,6\% dos elegíveis), mas não houve diferença conforme a idade, o sexo e a rede de ensino entre escolares avaliados e não avaliados. Ressalta-se que o processamento dos dados foi realizado por equipe de digitadores previamente treinados, sendo utilizado o sistema de dupla entrada de dados. Sendo assim, acredita-se que o rigor metodológico durante a investigação confere um alto grau de confiabilidade aos resultados, e houve um treinamento prévio da equipe de antropometristas 18 .

Por fim, ressalta-se que o objetivo deste estudo se limitou a avaliar o ambiente no entorno das escolas pela investigação entre tipos e distância dos pontos de venda de alimentos utilizados pelas famílias e sua associação com a prevalência de sobrepeso/obesidade em escolares. O estudo não teve por objetivo abordar a alimentação na escola, oferecida pelo Programa Nacional de Alimentação Escolar - PNAE, ou alimentos adquiridos nas cantinas escolares e/ou trazidos de casa, tampouco investigar outros importantes componentes da complexa e multicausal determinação do processo de sobrepeso/obesidade na população escolar.

Os dados encontrados evidenciam a existência de associação entre alguns tipos de pontos de venda de alimentos (supermercado e padaria) utilizados por famílias e a prevalência de sobrepeso/obesidade na população escolar. Tal fato demostra a importância do desenvolvimento de programas de intervenção e de apoio tanto à família quanto aos estabelecimentos que comercializam alimentos, objetivando reduzir a prevalência de sobrepeso/obesidade na população escolar.

Como proposta de continuidade na abordagem desta temática, sugere-se a realização de estudos sobre a relação entre os tipos de alimentos disponíveis nos pontos de venda de alimentos utilizados pela família e a prevalência de sobrepeso/ obesidade em escolares. 


\section{Resumen}

El estudio describe los puntos de venta de alimentos y su asociación con el sobrepeso/obesidad en escolares de Florianópolis, Santa Catarina, Brasil. Se trata de un estudio transversal con una muestra aleatoria de 2.506 escolares de las escuelas públicas $(n=19)$ y privadas $(n=11)$. El sobrepeso/obesidad se clasifica, en función de la OMS en 2007, con análisis ajustados y crudos que se realizaron mediante la regresión de Poisson. La prevalencia de sobrepeso/obesidad fue de un 34,2\%. En el sistema público el resultado fue de un 19,6\% sobrepeso y un 13,5\% obesidad. En el privado se observó un 22,4\% de sobrepeso y 11,1\% obesidad. En el primero se encontró una correlación entre el sobrepeso/obesidad y el consumo de bollería $(p=0,004)$. En las escuelas privadas se observó que los escolares de familias que habían utilizado el supermercado tenían un 26\% menos de sobrepeso/ obesidad que los niños en edad escolar que no utilizaron este punto de venta de alimentos $(p=0,003)$. En el momento del estudio existe una asociación entre el uso de algunos tipos de punto de venta de alimentos (supermercado y panadería) y la prevalencia de sobrepeso/obesidad en escolares.

Sobrepeso; Obesidad Pediátrica; Alimentación

\section{Colaboradores}

A. F. Motter e E. N. Correa foram responsáveis pela coleta, análise e interpretação dos dados, redação do artigo e aprovação final da versão a ser publicada. D. F. Andrade foi responsável pela assessoria estatística, revisão crítica relevante do conteúdo intelectual e aprovação final da versão a ser publicada. F. A. G. Vasconcelos foi responsável pela elaboração e coordenação do projeto, revisão crítica relevante do conteúdo intelectual e aprovação final da versão a ser publicada.

\section{Agradecimentos}

Os autores agradecem aos escolares e familiares que aceitaram participar do estudo e ao $\mathrm{CNPq}$ (processo no 483955/2011-6) por ter possibilitado e financiado esta pesquisa. A. F. Motter recebeu uma bolsa de estudos da Capes.

\section{Conflito de interesses}

Os autores do estudo declaram não existir conflito de interesses.

\section{Referências}

1. World Health Organization. Obesity and overweight. http://www.who.int/mediacentre/fact sheets/fs311/en/ (acessado em 13/Jul/2014).

2. World Health Organization. Fight childhood obesity to help prevent diabetes, say WHO \& IDF. http:// www.who.int/mediacentre/news/releases/2004/ pr81/en/index.html (acessado em 10/Fev/2013).

3. Instituto Brasileiro de Geografia e Estatística. Pesquisa de Orçamentos Familiares 2008-2009. Antropometria e sobrepeso e obesidade de crianças, adolescentes e adultos no Brasil. Rio de Janeiro: Instituto Brasileiro de Geografia e Estatística; 2010.

4. Assis MAA, Rolland-Cachera MF, Vasconcelos FAG, Bellisle F, Calvo MCM, Luna MEP, et al. Overweight and thinness in 7-9 year old children from Florianópolis, Southern Brazil: a comparison with a French study using a similar protocol. Rev Nutr 2006; 19:299-308.
5. Bernardo CO, Fernandes OS, Campos RMMB, Adami F, Vasconcelos FAG. Associação entre o índice de massa corporal de pais e de escolares de 7 a 14 anos de Florianópolis, SC, Brasil. Rev Bras Saúde Matern Infant 2010; 10:183-90.

6. Brug J, Kremers SP, Lenthe Fv, Ball K, Crawford D. Environmental determinants of healthy eating: in need of theory and evidence. Proc Nutr Soc 2008; 67:307-16.

7. Neutzling MB, Assunção CF, Malcon MC, Hallal PC, Menezes AM. Hábitos alimentares de escolares adolescentes de Pelotas, Brasil. Rev Nutr 2010; 23:379-88.

8. Glanz K, Kegler MC. Environments: theory, research and measures of the built environment. http://cancercontrol.cancer.gov/brp/constructs/ environment/environment.pdf (acessado em 15/ Ago/2014). 
9. Cimadon H, Geremia R, Pellanda LC. Hábitos alimentares e fatores de risco para aterosclerose em estudantes de Bento Gonçalves (RS). Arq Bras Cardiol 2010; 95:166-72.

10. Busick DB, Brooks J, Pernecky S, Dawson R, Petzoldt J. Parent food purchases as a measure of exposure and preschool-aged children's willingness to identify and taste fruit and vegetables. Appetite 2008; 51:468-73.

11. Epstein LH, Dearing KK, Handley EA, Roemmich JN, Paluch RA. Relationship of mother and child food purchases as a function of price: a pilot study. Appetite 2006; 47:115-8.

12. Pontes TE, Costa TF, Marum ABRF, Brasil ALD, Taddei JAAC. Orientação nutricional de crianças e adolescentes e os novos padrões de consumo: propagandas, embalagens e rótulos. Rev Paul Pediatr 2009; 27:99-105.

13. Spence JC, Cutumisu N, Edwards J, Raine KD, Smoyer-Tomic K. Relation between local food environments and obesity among adults. BMC Public Health 2009; 9:192.

14. Galvez MP, Hong L, Choi E, Liao L, Godbold J, Brenner B. Childhood obesity and neighborhood store availability in an inner city community. Acad Pediatr 2009; 9:339-43.

15. Leite FHM, Oliveira MA, Cremm EC, Abreu DSC, Maron LR, Martins PA. Oferta de alimentos processados no entorno de escolas públicas em área urbana. J Pediatr (Rio J.); 88:328-34.

16. de Onis M, Onyango AW, Borghi E, Siyam A, Nishida C, Siekmann J. Development of a WHO growth reference for school-aged children and adolescents. Bull World Health Organ 2007; 85:660-7.

17. Habicht J. Estandarización de métodos epidemiológicos cuantitativos sobre el terreno. Bol Oficina Sanit Panam 1974; 76:375-84.

18. Lohman TG, Roche AFE, Martorell R. Anthropometric standardization reference manual. Champaign: Human Kinetics Books; 1991.

19. Austin SB, Melly SJ, Sanchez BN, Patel A, Buka S, Gortmaker SL. Clustering of fast-food restaurants around schools: a novel application of spatial statistics to the study of food environments. Am J Public Health 2005; 95:1575-81.

20. Wall MM, Larson NI, Forsyth A, Van Riper DC, Graham DJ, Story MT, et al. Patterns of obesogenic neighborhood features and adolescent weight: a comparison of statistical approaches. Am J Prev Med 2012; 42:e65-75.

21. Brasil. Resolução CNS no 466, de 12 de dezembro de 2012. Diretrizes e normas regulamentadores de pesquisas envolvendo seres humanos. Diário Oficial da União 2013; 13 jun.

22. International Obesity Task Force. Childhood obesity report. London: International Obesity Task Force; 2004.

23. Wabitsch M, Moss A, Hauschild KK. Unexpected plateauing of childhood obesity rates in developed countries. BMC Public Health 2014; 12:17.
24. Oliveira SP, Muniz LB, Marliere CA, Freitas SN, Fonseca KZ, Carvalho LR, et al. Hábitos de compra de alimentos da população de Ouro Preto (Minas Gerais). Segurança Alimentar e Nutricional 2005; 12:1-9.

25. Zenk SN, Schulz AJ, Israel BA, James SA, Bao S, Wilson ML. Neighborhood racial composition, neighborhood poverty, and the spatial accessibility of supermarkets in metropolitan Detroit. Am J Public Health 2005; 95:660-7.

26. Wang MC, Gonzalez AA, Ritchie LD, Winkleby MA. The neighborhood food environment: sources of historical data on retail food stores. Int J Behav Nutr Phys Act 2006; 3:15.

27. Popkin BM. Global nutrition dynamics: the world is shifting rapidly toward a diet linked with noncommunicable diseases. Am J Clin Nutr 2006; 84:289-98.

28. Hutchinson PL, Bodor JN, Swalm CM, Rice JC, Rose D. Neighbourhood food environments and obesity in southeast Louisiana. Health Place 2012; 18:854-60.

29. Laraia BA, Siega-Riz AM, Kaufman JS, Jones SJ. Proximity of supermarkets is positively associated with diet quality index for pregnancy. Prev Med 2004; 39:869-75.

30. Wang R, Shi L. Access to food outlets and children's nutritional intake in urban China: a difference-indifference analysis. Ital J Pediatr 2012; 38:30.

31. An R, Sturm R. School and residential neighborhood food environment and dietary intake among California children and adolescents. Am J Prev Med 2012; 42:129-35.

32. Howard PH, Fitzpatrick M, Fulfrost B. Proximity of food retailers to schools and rates of overweight ninth grade students: an ecological study in California. BMC Public Health 2011; 11:1-8.

33. Instituto Brasileiro de Geografia e Estatística. Comissão Nacional de Classificação. http://www. cnae.ibge.gov.br $/$ classe. asp? codclasse $=47211 \&$ co dgrupo $=472 \&$ CodDivisao $=47 \&$ CodSecao $=$ G $\&$ Tabe laBusca=CNAE_200@CNAE\%202.0 (acessado em 10/Mai/2014).

34. Leung CW, Laraia BA, Kelly M, Nickleach D, Adler NE, Kushi LH, et al. The influence of neighborhood food stores on change in young girls' body mass index. Am J Prev Med 2011; 41:43-51.

35. Sturm R, Datar A. Body mass index in elementary school children, metropolitan area food prices and food outlet density. Public Health 2005; 119:1059-68.

36. Cheadle A, Psaty BM, Curry S, Wagner E, Diehr P, Koepsell T, et al. Comparisons between the grocery store environment and individual dietary practices. Prev Med 1991; 20:250-61.

37. Duran ACFL. Ambiente alimentar urbano em São Paulo, Brasil: avaliação, desigualdades e associação com consumo alimentar [Tese de Doutorado]. São Paulo: Faculdade de Saúde Pública, Universidade de São Paulo; 2013. 
38. Jekanowski MD, Binkley JK, Eales J. Convenience, accessibility, and the demand for fast food. Journal of Agricultural and Resource Economics 2001; 26:58-74.

39. Hattori A, An R, Sturm R. Neighborhood food outlets, diet, and obesity among California adults, 2007 and 2009. Prev Chronic Dis 2013; 10:E35.

40. Wang N, Xu F, Zheng LQ, Zhang XG, Li Y, Sun GZ, et al. Effects of television viewing on body fatness among Chinese children and adolescents. Chin Med J (Engl) 2012; 125:1500-3.

41. Caughy MO, Leonard T, Beron K, Murdoch J. Defining neighborhood boundaries in studies of spatial dependence in child behavior problems. Int J Health Geogr 2013; 12:24.
42. Laska MN, Borradaile KE, Tester J, Foster GD, Gittlelsohn J. Healthy food availability in small urban food stores: a comparison of four US cities. Public Health Nutr 2010; 13:1031-5.

43. Bodor JN, Ulmer VM, Dunaway LF, Farley TA, Rose D. The Rationale behind small food store interventions in low-income urban neighborhoods: insights from New Orleans. J Nutr 2010; 140:1185-8.

44. Gittelsohn J, Rowan M, Gadhoke P. Interventions in small food stores to change the food environment, improve diet, and reduce risk of chronic disease. Prev Chronic Dis 2012; 9:E59.

Recebido em 27/Jun/2014

Versão final reapresentada em 02/Set/2014

Aprovado em 24/Set/2014 